\title{
Phonon-Assisted Electron Hopping Conduction in the Uranium Doped One-Dimensional Antiferromagnet $\mathrm{Ca}_{2} \mathrm{CuO}_{3}$
}

\author{
Phung Quoc Thanh ${ }^{1,2}$, Seong-Cho $\mathrm{Yu}^{2 *}$, and Hoang Nam Nhat ${ }^{1}$ \\ ${ }^{1}$ Center for Materials Science, College of Science, Vietnam National University Hanoi, 334-Nguyen Trai, Hanoi, Vietnam \\ ${ }^{2}$ Department of Physics, Chungbuk National University, Cheongju 361-763, Korea
}

(Received 25 September 2008, Received in final form 1 December 2008, Accepted 2 December 2008)

\begin{abstract}
The authors studied the conduction mechanism in an uranium doped low dimensional magnetic system $\mathrm{Ca}_{2} \mathrm{CuO}_{3}$. This system exhibits the $\mathrm{S}=1 / 2$ quasi $1 \mathrm{D}$ antiferromagnetic chains of $-\mathrm{Cu}-\mathrm{O}$ - with strong magnetic coupling, and demonstrates continuous semiconductor-like behavior with constant covalent insulator character. This paper identifies the conduction is due to thermally activated phonon-assisted electron hopping between dopant uranium sites. The parameter á, the characteristic for hopping probability, was determined to be 0.18 $\AA^{-1}$. This value manifests a relatively stronger hopping probability for $\mathrm{Ca}_{2} \mathrm{CuO}_{3}$ as compared with other uranium doped ceramics.
\end{abstract}

Keywords : uranium, phonon-assisted, conduction, antiferromagnet

\section{Introduction}

Recently, uranium-doping studies in high $T_{c}$ superconductors, pyroelectric ceramics, and oxide based optical materials have shown significant improvements in the desired properties of these materials, such as, higher current densities, better resistance ranges, and optical transitions in the visible region [1-5]. Furthermore, it has been shown that doping with a small amount of the oxygen-rich uranium oxide $\mathrm{U}_{3} \mathrm{O}_{7}$ in the strongly anisotropic $\mathrm{S}=1 / 2$ quasi $1 \mathrm{D}$ antiferromagnetic system, induced a relatively large change in its resistance but an unchanged covalent insulator state [6]. Pure $\mathrm{Ca}_{2} \mathrm{CuO}_{3}$ possesses an extremely low ordered moment $\left(\approx 0.05 \mu_{\mathrm{B}}\right)$ and a reduced Néel temperature $(9 \mathrm{~K})$ and its in-chain -Cu-Oantiferromagnetic interaction is much larger than its interchain coupling $\left(J_{\|} \approx 0.2 \mathrm{eV}, J_{\perp} \approx 0.01 \mathrm{meV}\right)$ [7]. An $a b$ initio study revealed only a small gap between HOMO (Highest Occupied Molecular Orbitals) and LUMO (Lowest Unoccupied Molecular Orbitals), in this case between $\mathrm{Cu}(3 \mathrm{~d})-\mathrm{O}(2 \mathrm{p})$ bonding and antibonding molecular orbitals. Thus, the activation energy from the insulation ground state to the conduction band is expected to be small (of the order $\mathrm{meV}$ ), but the localization of HOMO's electrons

*Corresponding author: Tel: +82-43-261-2269

Fax:+82-43-275-6416, e-mail: scyu@chungbuk.ac.kr is a key factor that maintains the insulating status of $\mathrm{Ca}_{2} \mathrm{CuO}_{3}$. In Fig. 1 we illustrate the HOMO and LUMO obtained by $a b$ initio calculation for the model cluster $\mathrm{Ca}_{2} \mathrm{Cu}_{4} \mathrm{O}_{10}$ in the ideal $\mathrm{Ca}_{2} \mathrm{CuO}_{3}$ structure. The calculation was carried out using Gaussian 2003 software [8] and was based on the Density Functional Theory using the hybrid functionals B3LYP with the STO-3G wave function basic set. The localization of HOMO mechanically explains the covalent-insulator characteristics of bulk $\mathrm{Ca}_{2} \mathrm{CuO}_{3}$. Furthermore, despite its low thermal activation energy $E_{a}(<0.2 \mathrm{eV})$, the resistance of material is of order $100 \mathrm{M} \Omega$ With increasing temperature, the number of electrons that gain sufficient energy to jump across the barrier grows exponentially, and thus, the macroscopic manifestation of $\mathrm{Ca}_{2} \mathrm{CuO}_{3}$ is semiconductor-like. Three different models are available for fitting $\mathrm{R}(\mathrm{T})$ data: the band-gap model $\left(\ln (\rho) \propto E_{a} / k_{B} T\right)$, the small polaron hopping model $\left(\ln (\rho / T) \propto W / k_{B} T\right)$ and the variable-range hopping model (with or without magnetic localization [9], $\left.\log \left(\rho / \rho_{\infty}\right) \propto\left(T_{0} T\right)^{-1 / 4}\right)$. Whereas the band-gap model yielded an acceptable result, the other two offered the worse least-square figure-of-merits.

The recently proposed percolative conduction grain boundary system as a fractal conduction medium $\left(1 / T^{n}\right.$ law) [10] produced a good result, but the physics of this model do not comply with the covalent-insulator characteristics of the samples studied, and thus, we can not be 
considered here. When uranium is introduced into the $\mathrm{Ca}_{2} \mathrm{CuO}_{3}$ structure, there is enough room to accommodate uranium atoms in $\mathrm{Ca}^{2+}$ sites along the $c$ axis $[1,6]$, but uranium might also be located at interstitial regions on single crystals boundaries. The recently obtained Raman scattering data of doped samples support this argument, since this data was almost independent of uranium content [11]. However, for the analysis, we only needed uranium atoms to be homogeneously distributed in the bulk matrix. Under this presumption, the linear distance between dopant atoms may be estimated according to the classical law:

$$
R=a z^{-1 / 3}
$$

where $a$ is the lattice constant and $z$ is the concentration of dopant uranium. The lattice (Immm) in our case was not cubic ( $a=3.25, b=3.77$ and $c=12.24 \AA)$ and the value of $a$ in (1) should be the average linear distance between the two closest dopant sites, i.e. $3.25 \AA$ along the $a$ axis. This linear distance $R$ is an exponential factor for the probability of electron hopping between dopant sites:

$$
p=v \exp (-\alpha R)
$$

where $v$ is a factor dependent on phonon frequency and $\alpha$ is a parameter of exponential distribution and a characteristic of each dopant system. In systems where conduction is dominated by the thermally activated hopping of electrons, the amplitude of $d c$-conductivity should be proportional to hopping probability, so may be given as $\sigma_{0}=k v \exp \left(-\alpha a z^{-1 / 3}\right)$, where $k$ is a scale constant.

Incorporating this into the expression $\sigma=\sigma_{0} \exp \left(-E_{a} /\right.$ $k_{\mathrm{B}} T$ ) for the $d c$-conductivity, we obtain:

$$
\rho=1 / \sigma=A \exp \left(\alpha a z^{-1 / 3}+E_{d} / k_{\mathrm{B}} T\right)
$$

where $\rho$ is the $d c$-resistivity, $k_{\mathrm{B}}$ the Boltzmann constant, $E_{a}$ the activation energy and $A=1 / k v$. The alternative casting is the log-log form:

$$
\ln \rho=\ln A+\alpha a z^{-1 / 3}+E_{a} / k_{\mathrm{B}} T
$$

It is worth noting that while the fitting of $\ln \rho$ against $1 /$ $T$ yields the activation energy $E_{a}$ in the slope, fitting at fixed $T$ of $\ln \rho$ against $z^{-1 / 3}$ yields $\alpha a$ in the slope. This provides an efficient means of estimating the parameter $\alpha$, which is a characteristic of different hopping systems. Since the factor $A$ in equation (3) is inversely related to phonon frequency which is corresponds to a larger hopping probability. For this reason, equation (3) illustrates the hopping mechanism assisted by a phonon. The most important outcome of (3)-(4) follows from the fact that the final conductivity depends only on concentration of dopant and not on the material composition, which provides

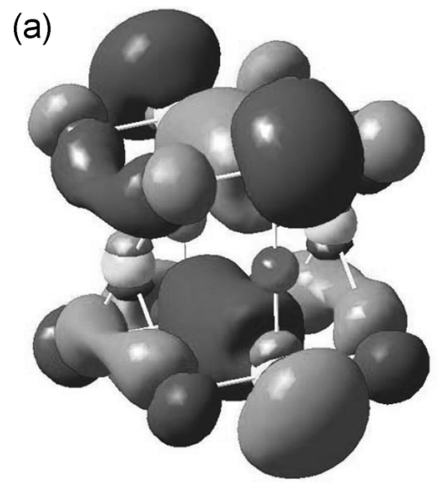

(b)

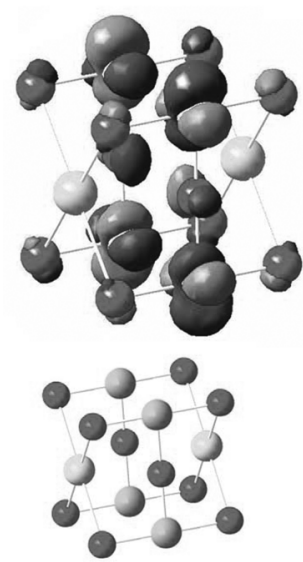

Fig. 1. (Color online) $A b$ initio calculation of $\mathrm{HOMO}$ (a) and LUMO (b) for the $\mathrm{Ca}_{2} \mathrm{Cu}_{4} \mathrm{O}_{10}$ cluster (c) in the ideal $\mathrm{Ca}_{2} \mathrm{CuO}_{3}$ structure (DFT/B3LYP/STO-3G [12]). In (c): the red (dark) balls represent oxygen, the yellow (gray) balls calcium, and the pink (half-dark) balls copper. In (a) and (b): the gray variations of molecular orbitals (MOs) depict orbital symmetry differences. In HOMO, apical oxygens (i.e. $\mathrm{O}(1)$ atoms lying along axis $c$ ) do not contribute to $\mathrm{Cu}$ bonding, and the in-chain oxygens (i.e. the $\mathrm{O}(2)$ atoms lying along axis $b$ ) appear to have only localized MOs with nearest neighbors. However, in HOMO, $\mathrm{Cu}$ atoms form direct $\mathrm{Cu}-\mathrm{Cu}$ couplings (bonds?) (along axis $a$ ). HOMO does not contribute to $\mathrm{Cu}-\mathrm{O}$ chain coupling. The $\mathrm{Ca}_{2} \mathrm{Cu}_{4} \mathrm{O}_{10}$ cluster has 122 bonding MOs in which HOMO is orbital no.122, LUMO is orbital no.123, of the total 148 MOs. As seen in (b), LUMO consists of almost separate atomic orbitals (AOs) that do not appear to involve $\mathrm{Ca}$ atoms.

a means of comparing the conductivities of various systems despite differences in their chemical contents.

For $\mathrm{Ca}_{2} \mathrm{CuO}_{3}: \mathrm{U}_{z}(z=0.00,0.005,0.02,0.025,0.05)$, experimental resistivity data were taken from [6]. These data were collected using the standard four electrode technique using equipment from Bio-Rad that was capable of detecting pico-ampere currents; it was cooled down using a software-controlled closed-cycle He refrigerator. The compounds themselves were prepared using a modification of a previously described sol-gel method [5], which was used to prepare highly pure homogeneous single-phase $\mathrm{Ca}_{2} \mathrm{CuO}_{3}$ powder. Fig. 2 shows the results of $1 / \mathrm{T}$ fit, whereby we obtained $E_{a}=0.19 \mathrm{eV}$ for the undoped sample and $E_{a}=0.035 \mathrm{eV}$ for doped samples. These values are substantially smaller than the $1.70 \mathrm{eV}$ obtained via optical measurements [12], but correspond well to $0.18 \mathrm{eV}$ reported in [13]. Uranium doping seems to substantially reduce the energy gap between the insulation and conduction bands. The value $E_{a}=0.035 \mathrm{eV}$ agrees reasonably well with the HOMO/LUMO gap of $0.046 \mathrm{eV}$. From $a b$ initio calculation, both 0.19 and $0.035 \mathrm{eV}$ fall within the $0.46 \mathrm{eV}$ range between the LUMO and HUMO 


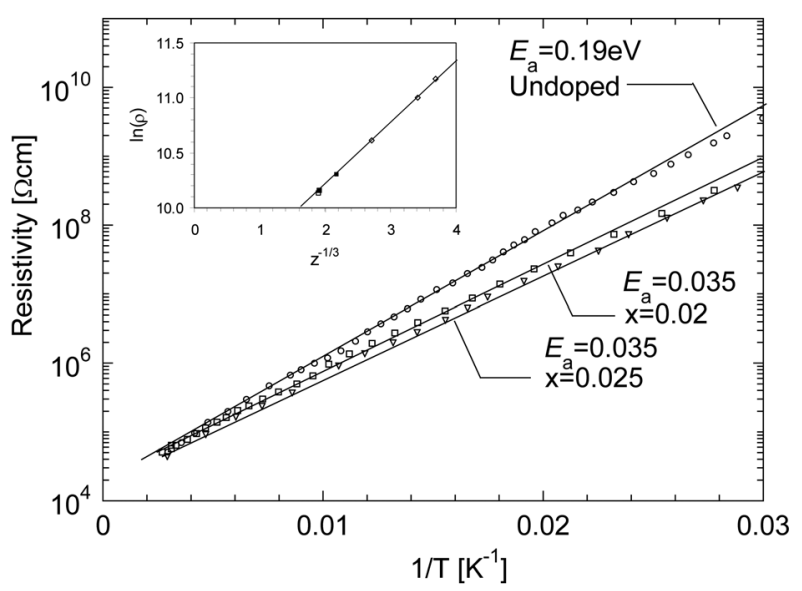

Fig. 2. Fit results for uranium doped $\mathrm{Ca}_{2} \mathrm{CuO}_{3}$ according to equation (4). The inset shows the fit result for hopping parameter $\alpha$, in which the dark squares denote forecast values and blank squares denote actual cases.

(Highest Unoccupied MO). Thus, for the ideal $\mathrm{Ca}_{2} \mathrm{CuO}_{3}$ structure if we consider $\mathrm{HOMO}$ the insulation ground state, the conduction band may be located somewhere between LUMO and HUMO. The ab initio result for the small cluster $\mathrm{Ca}_{2} \mathrm{Cu}_{4} \mathrm{O}_{10}$ did not explain the large gap 1.70 eV obtained by optical measurements; optical excitation may be associated with electron-phonon coupling via some charge transfer process along the -Cu-O- chain. Possibly, larger clusters must be taken into account during studies of optical processes. The high resisitivity and the small activation energy make $\mathrm{U}$-doped $\mathrm{Ca}_{2} \mathrm{CuO}_{3}$ samples typical covalent insulators. For comparison purposes, it should be noted that the resistivity of $(\mathrm{Mg}, \mathrm{Nb})-\mathrm{PZT}$ ceramics is $47.8 \mathrm{M} \Omega \mathrm{cm}$, while their activation energy is $0.34 \mathrm{eV}$ [1]. U-doped $\mathrm{Ca}_{2} \mathrm{CuO}_{3}$ had a resistivity of $>100 \mathrm{M} \Omega \mathrm{cm}$, but and activation energy of $0.035 \mathrm{eV}$. In the inset in Fig. 2 we show the fitting for the characteristic hopping probability parameter a, the slope of which gave the result $\mathrm{a}=$ $0.18 \AA^{-1}$. This slope is independent of temperature $T$ and phonon frequency $v$ and depends only on the linear distance between dopant sites $R$, i.e. on the lattice constant a. The equation (4) was used in [1] to determine the constant $\alpha=0.63 \AA^{-1}$ for U-doped ( $\left.\mathrm{Mg}, \mathrm{Nb}\right)$ PZT ceramics (U concentration was $c a .0 .005 \mathrm{~mol} \mathrm{U} / 1 \mathrm{~mol} \mathrm{~Pb}$ ).

Fig. 3 shows the dependence of the normalized "phonon-frequency independent" probability $p \exp (a) / v$ on dopant concentration $z$ for two cases, i.e., $\alpha=0.18 \AA^{-1}$ (U-doped $\mathrm{Ca}_{2} \mathrm{CuO}_{3}$ system) and $\alpha=0.63 \AA^{-1}$ (U-doped $\mathrm{Mg}, \mathrm{Nb}-\mathrm{PZT}$ system [1]). At a doping concentration of $z=$ 0.05 , the curve for $\alpha=0.18 \AA^{-1}$ showed a 15 times greater hopping probability than that of $\alpha=0.63 \AA^{-1}$. The ratio of non-normalized probabilities was even higher $(\sim 150$ times greater if the phonon frequencies are considered as equal).

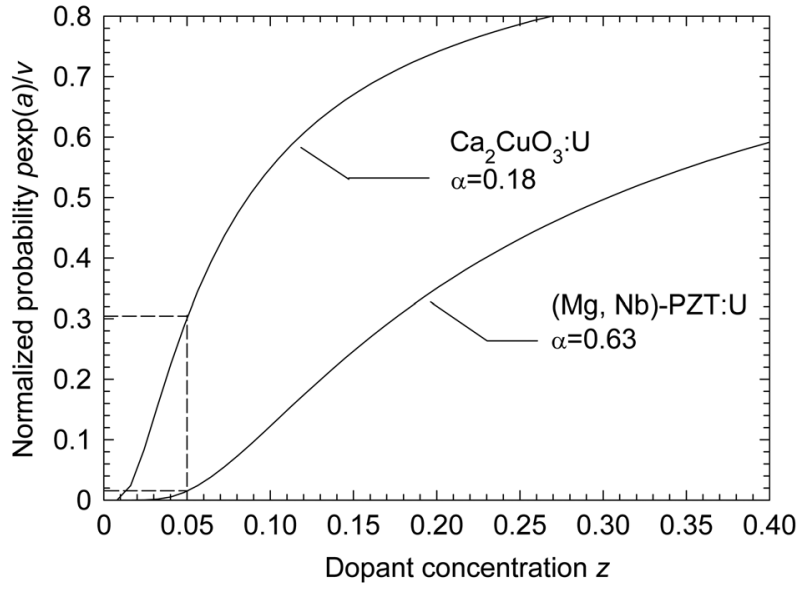

Fig. 3. The normalized hopping probabilities of two different uranium doped systems: $\mathrm{Ca}_{2} \mathrm{CuO}_{3}$ with $\alpha=0.18 \AA^{-1}$ and $(\mathrm{Mg}$, $\mathrm{Nb})$-PZT with $\alpha=0.63 \AA^{-1}[1]$.

This effect is due purely to differences in the linear distances between dopant sites $R$. For doping concentration $z$ from 0.005 to 0.05 in $\mathrm{Ca}_{2} \mathrm{CuO}_{3}: \mathrm{U}_{z}, R$ reduced from 19.0 to $8.8 \AA$ (the smaller value is about $2.7 a$ ). To identify the role of uranium in electron hopping from the insulation ground state to the conduction band, the HOMO and LUMO of U-doped cases should be constructed, at least for the modified cluster $\mathrm{Ca}_{1} \mathrm{U}_{1} \mathrm{Cu}_{4} \mathrm{O}_{10}$. However, this could not be done due to the absence of a basic wave function set for atoms with atomic number greater than 54 (uranium no. is 92). Most of the currently available basic sets are applicable only for $\mathrm{H}-\mathrm{Cl}$ (almost all sets) except the STO$3 \mathrm{G}$ set, which extends to $\mathrm{H}-\mathrm{Xe}$. This issue requires further consideration.

The authors are grateful to the Asian Research Center (Vietnam National University) (\#QG-TD-2009), and International Scholar Exchange Fellowship for the Academic Year of 2008-2009 of the Korea Foundation for Advanced Studies (KFAS) for financial support.

\section{References}

[1] S. B. Stringfellow, S. Gupta, C. Shaw, J. R. Alcock, and R. W. Whatmore, J. of the Eur. Cer. Soc. 22, 573 (2002).

[2] R. Weinstein, US Patent 6083885 (2000) and R. Weinstein, and R. P. Sawh, Supercond. Sci. Technol. 15, 1474 (2002).

[3] N. Hari Babu, M. Kambara, Y. Shi, D. A. Cardwell, C. D. Tarrant, and K.R. Schneider, IEEE Trans. on Appl. Supercond. 13, 3147 (2003).

[4] M. Eder and G. Gritzner, Supercond. Sci. Technol. 13, 1302 (2000).

[5] D. C. Huynh, D. T. Ngo, and N. N. Hoang, J. of Phys: Cond. Matters 19, 106215 (2007). 
[6] N. N. Hoang, D. C. Huynh, T. T. Nguyen, D. T. Ngo, D. T. Nguyen, A. Fennie, and Nguyen Chau, Applied Phys. A 92, 715-725 (2008), DOI: 10.1007/s00339- 008-4631$\mathrm{y}$.

[7] K. Yamada, J. Wada, S. Hosoya, Y. Endoh, S. Noguchi, S. Kawamata, and K. Okuda, Physica C 253, 135 (1995).

[8] Gaussian 03, Revision B.03, M. J. Frisch, G. W. Trucks, H. B. Schlegel, G. E. Scuseria, M. A. Robb, J. R. Cheeseman, J. A. Montgomery, Jr., T. Vreven, K. N. Kudin, J. C. Burant, J. M. Millam, S. S. Iyengar, J. Tomasi, V. Barone, B. Mennucci, M. Cossi, G. Scalmani, N. Rega, G. A. Petersson, H. Nakatsuji, M. Hada, M. Ehara, K. Toyota, R. Fukuda, J. Hasegawa, M. Ishida, T. Nakajima, Y. Honda, O. Kitao, H. Nakai, M. Klene, X. Li, J. E. Knox, H. P. Hratchian, J. B. Cross, C. Adamo, J. Jaramillo, R. Gomperts, R. E. Stratmann, O. Yazyev, A. J. Austin, R. Cammi, C. Pomelli, J. W. Ochterski, P. Y. Ayala, K. Morokuma, G. A. Voth, P. Salvador, J. J. Dannenberg, V. G. Zakrzewski, S. Dapprich, A. D. Daniels, M. C. Strain,
O. Farkas, D. K. Malick, A. D. Rabuck, K. Raghavachari, J. B. Foresman, J. V. Ortiz, Q. Cui, A. G. Baboul, S. Clifford, J. Cioslowski, B. B. Stefanov, G. Liu, A. Liashenko, P. Piskorz, I. Komaromi, R. L. Martin, D. J. Fox, T. Keith, M. A. Al-Laham, C. Y. Peng, A. Nanayakkara, M. Challacombe, P. M. W. Gill, B. Johnson, W. Chen, M. W. Wong, C. Gonzalez, and J. A. Pople, Gaussian, Inc., Pittsburgh PA, 2003.

[9] M. Viret, L. Ranno, and J. M. D. Coey, Journal of Applied Physics 81(8), 4964 (1997).

[10] N. N. Hoang, D. C. Huynh, and M. H. Phan, Solid State Commun. 139, 456 (2006).

[11] K. Maiti, D. D. Sarma, T. Mizokawa, and A. Fujimori, Phys. Rev. B 57, 1572 (1998).

[12] D. R. Lines, M. T. Weller, D. B. Currie, and D. M. Ogborne, Mater. Res. Bull. 26, 323 (1991).

[13] N. N. Hoang, T. H. Nguyen, and Chau Nguyen, J. Appl. Phys. 103, 093524 (2008). 